\title{
REVISED Automated cell tracking using StarDist and TrackMate
}

\section{[version 2; peer review: 3 approved]}

\author{
Elnaz Fazeli (iD1, Nathan H. Roy², Gautier Follain 3,4, Romain F. Laine (iD) 5,6, \\ Lucas von Chamier ${ }^{5}$, Pekka E. Hänninen ${ }^{1}$, John E. Eriksson ${ }^{3,4}$, \\ Jean-Yves Tinevez (iD)7, Guillaume Jacquemet (D) 3,4
}

\footnotetext{
${ }^{1}$ Laboratory of Biophysics, Institute of Biomedicine, Faculty of Medicine, University of Turku, Turku, Finland

${ }^{2}$ Department of Pathology and Laboratory Medicine, Children's Hospital of Philadelphia Research Institute, Philadelphia, PA 19104, USA

${ }^{3}$ Turku Bioscience Centre, University of Turku and Åbo Akademi University, Turku, Finland

${ }^{4}$ Cell Biology, Faculty of Science and Engineering, Åbo Akademi University, Turku, Finland

${ }^{5}$ MRC-Laboratory for Molecular Cell Biology, University College London, London, UK

${ }^{6}$ The Francis Crick Institute, London, UK

${ }^{7}$ Image Analysis Hub, Institut Pasteur, Paris, France
}

V2 First published: 28 Oct 2020, 9:1279

https://doi.org/10.12688/f1000research.27019.1

Latest published: 21 Dec 2020, 9:1279

https://doi.org/10.12688/f1000research.27019.2

\section{Abstract}

The ability of cells to migrate is a fundamental physiological process involved in embryonic development, tissue homeostasis, immune surveillance, and wound healing. Therefore, the mechanisms governing cellular locomotion have been under intense scrutiny over the last 50 years. One of the main tools of this scrutiny is live-cell quantitative imaging, where researchers image cells over time to study their migration and quantitatively analyze their dynamics by tracking them using the recorded images. Despite the availability of computational tools, manual tracking remains widely used among researchers due to the difficulty setting up robust automated cell tracking and large-scale analysis. Here we provide a detailed analysis pipeline illustrating how the deep learning network StarDist can be combined with the popular tracking software TrackMate to perform 2D automated cell tracking and provide fully quantitative readouts. Our proposed protocol is compatible with both fluorescent and widefield images. It only requires freely available and open-source software (ZeroCostDL4Mic and Fiji), and does not require any coding knowledge from the users, making it a versatile and powerful tool for the field. We demonstrate this pipeline's usability by automatically tracking cancer cells and T cells using fluorescent and brightfield images. Importantly, we provide, as supplementary information, a detailed step-by-step protocol to allow researchers to implement it with their images.

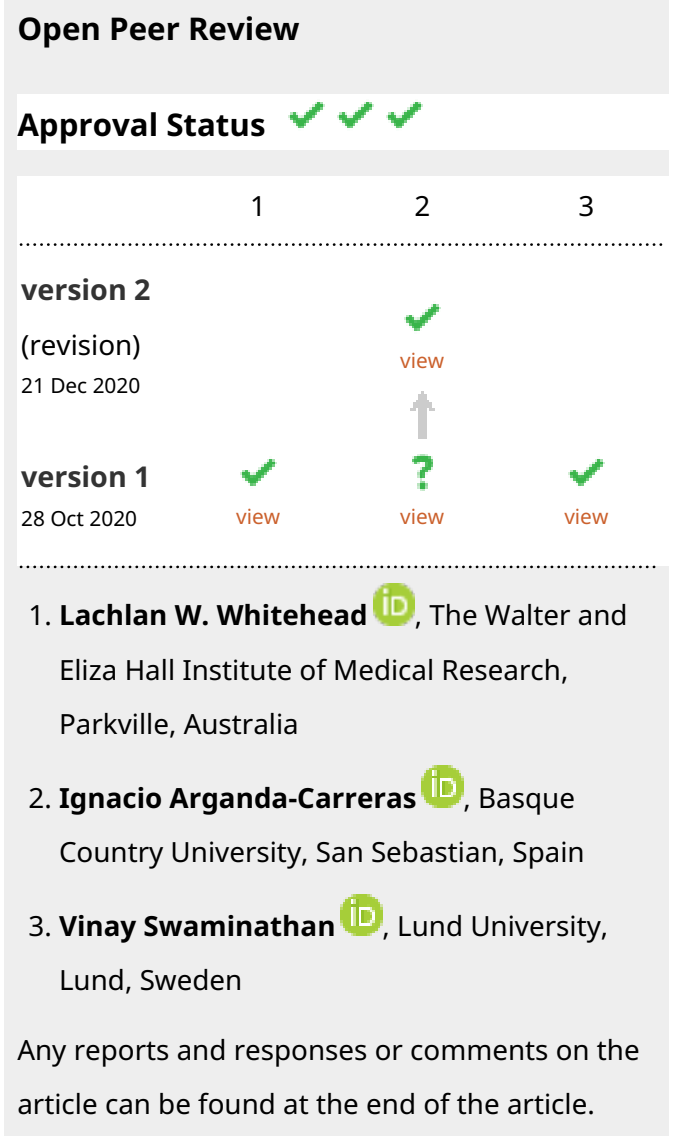




\section{Keywords}

Cell migration, Image analysis, StarDist, TrackMate, Deep-learning,

Automated tracking

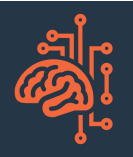

This article is included in the Artificial

Intelligence and Machine Learning gateway.

This article is included in the NEUBIAS - the

Bioimage Analysts Network gateway.

Corresponding author: Guillaume Jacquemet (guillaume.jacquemet@abo.fi)

Author roles: Fazeli E: Formal Analysis, Investigation, Software, Visualization, Writing - Original Draft Preparation, Writing - Review \& Editing; Roy NH: Data Curation, Investigation, Writing - Review \& Editing; Follain G: Data Curation, Investigation, Writing - Review \& Editing; Laine RF: Methodology, Software, Writing - Review \& Editing; von Chamier L: Methodology, Software, Writing - Review \& Editing; Hänninen PE: Writing - Review \& Editing; Eriksson JE: Writing - Review \& Editing; Tinevez JY: Methodology, Software, Writing Review \& Editing; Jacquemet G: Conceptualization, Data Curation, Formal Analysis, Funding Acquisition, Investigation, Methodology, Project Administration, Resources, Software, Supervision, Validation, Visualization, Writing - Original Draft Preparation, Writing - Review \& Editing

Competing interests: No competing interests were disclosed.

Grant information: This work was supported by grants awarded by the Academy of Finland (G.J.), the Sigrid Juselius Foundation (G.J.), Åbo Akademi University Research Foundation (CoE CellMech; G.J.) and by Drug Discovery and Diagnostics strategic funding to Åbo Akademi University (G.J.). G.F. was supported by National Cancer Center Finland (FICAN). NHR acknowledges support from the Cancer Research Institute and NIH T32 AR007442. R.F.L. acknowledges the support of the MRC Skills development fellowship (MR/T027924/1). The funders had no role in study design, data collection and analysis, decision to publish, or preparation of the manuscript.

Copyright: $\odot 2020$ Fazeli E et al. This is an open access article distributed under the terms of the Creative Commons Attribution License, which permits unrestricted use, distribution, and reproduction in any medium, provided the original work is properly cited.

How to cite this article: Fazeli E, Roy NH, Follain G et al. Automated cell tracking using StarDist and TrackMate [version 2; peer review: 3 approved] F1000Research 2020, 9:1279 https://doi.org/10.12688/f1000research.27019.2

First published: 28 Oct 2020, 9:1279 https://doi.org/10.12688/f1000research.27019.1 


\section{REVISED Amendments from Version 1}

- The size of the training dataset is now included in the main text

- A sentence indicating that the pipeline is suitable to track dividing cells has is also now included

Any further responses from the reviewers can be found at the end of the article

\section{Introduction}

The study of cell motility typically involves recording cell behavior, using live-cell imaging, and tracking their movement over time $^{1,2}$. To enable the analysis of such data, various software solutions have been developed ${ }^{3-9}$. However, despite the availability of these computational tools, manual tracking remains widely used among researchers due to the difficulty in setting up fully automated cell tracking analysis pipelines. Automated tracking pipelines share a typical workflow that starts with a segmentation strategy that identifies the objects to track in each image. Tracking algorithms are then used to link these objects between frames. One challenging aspect of an automated tracking pipeline is often achieving an accurate segmentation of the objects to track. One option to facilitate cell segmentation is to label their nuclei, using fluorescent dyes or protein markers. Nuclei can then be automatically segmented using intensity-based thresholding. However, this approach tends to become inaccurate when images are noisy or when the cells to track are very crowded $^{10}$. Deep Learning approaches have demonstrated their robustness against these two issues ${ }^{11}$. In this work, we present a new analysis workflow that builds upon a Deep Learning segmentation tool and a cell tracking tool to achieve robust cell tracking in cell migration assays. We combine StarDist, a powerful deep learning-based segmentation tool, and TrackMate, a user-friendly tracking tool, into a tracking pipeline that can be used without requiring expertise in or specialized hardware for computing (Figure 1) ${ }^{12-15}$.

\section{Methods}

Pipeline

The use of deep learning networks, such as StarDist, often requires the user to train or retrain a model using their images. While high-quality StarDist pre-trained models are readily available, they are likely to underperform when used on different data with, e.g., different staining, noise, and microscope type $^{15}$. To train StarDist models, we took advantage of the ZeroCostDL4Mic platform, allowing researchers to train (and

\section{Train a StarDist model}

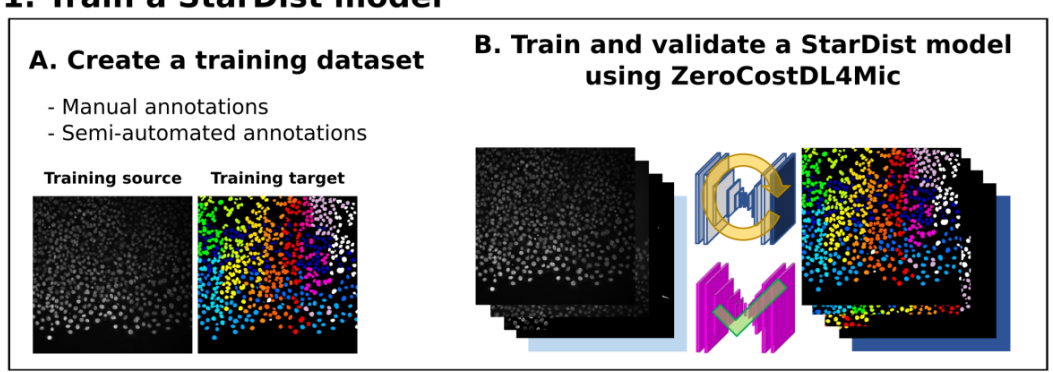

\section{Generate "Tracking files" using a StarDist model}

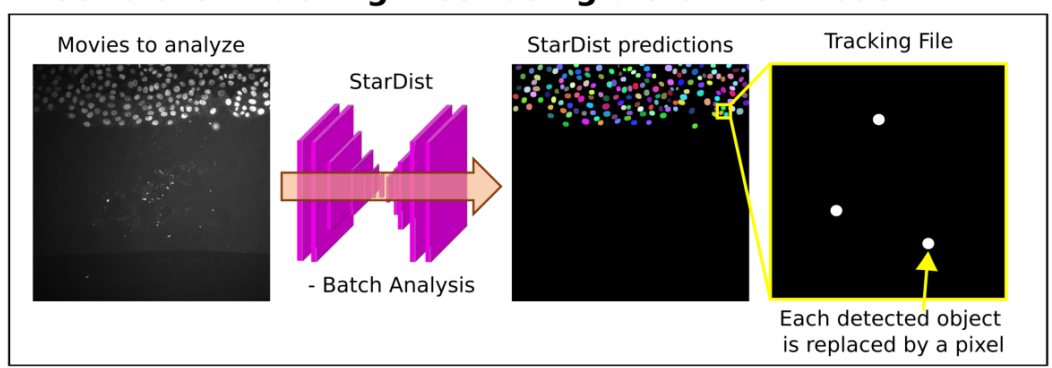

\section{Tracking the "Tracking files" using TrackMate}

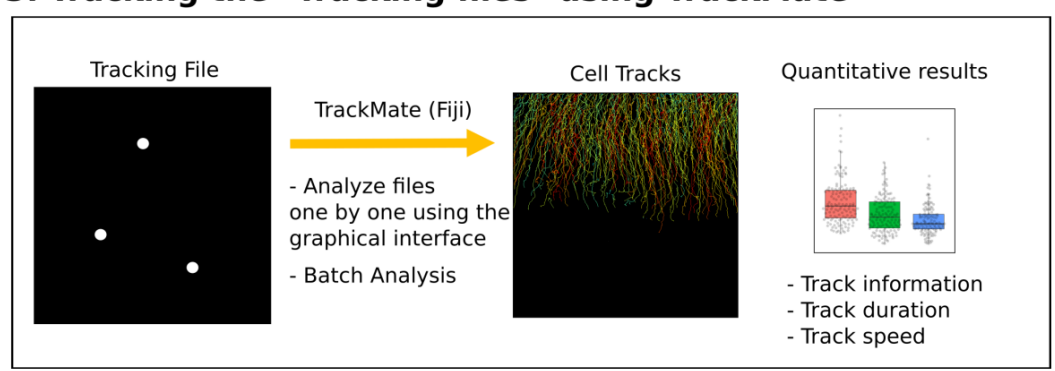

Figure 1. Workflow depicting how StarDist and TrackMate can be combined to track cells automatically. 
retrain), validate, and use deep learning networks ${ }^{15}$. Importantly, the ZeroCostDL4Mic StarDist 2D notebook can directly output a file containing all the nuclei's geometric center coordinates (named tracking files), that can be used as input for TrackMate (Figure 1). Therefore, our proposed pipeline can be divided into three parts (Figure 1; Extended data ${ }^{16}$ ). 1) First, a StarDist model is trained using the ZeroCostDL4Mic platform. This part needs to be performed only once for each type of data. 2) Second, the trained StarDist model is used to segment the object to track and generate Tracking files. 3) Finally, the tracking files can be used in TrackMate to track the identified objects.

Training a StarDist model requires a set of images and their corresponding masks (Figure 1 and Figure 2). Generating a training dataset is by far the most time-consuming part of
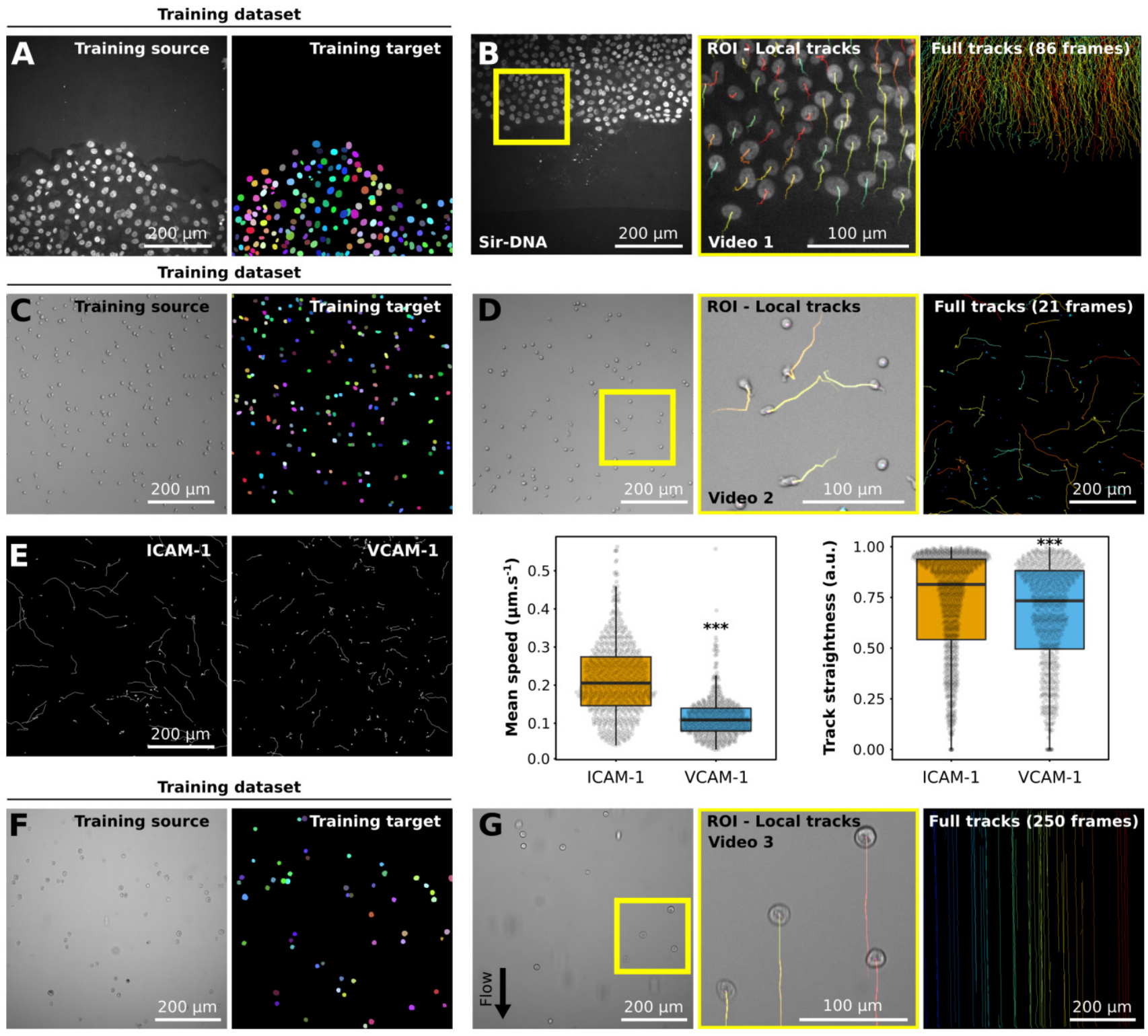

Figure 2. Example of datasets analyzed using StarDist and TrackMate. (A, B) Migration of MCF10DCIS.com, labeled with Sir-DNA, recorded using a spinning disk confocal microscope and automatically tracked. Examples of images used to train StarDist (A), and an example of results obtained using automated tracking are displayed (B, Video 1). The yellow square indicates a magnified ROI, where the local track of each nucleus is displayed. The full cell tracks are displayed on the left. Tracks are color-coded as a function of their maximum instantaneous velocity (blue slow, red fast tracks). (C-E) Migration of activated T cell plated on VCAM-1 or ICAM-1, recorded using a brightfield microscope and automatically tracked. Examples of images used to train StarDist (C) and an example of results obtained using automated tracking are displayed (D, Video 2). (E) Comparison of the migration of activated T cells on VCAM-1 or ICAM-1. Track mean speed and track straightness were quantified. Data are displayed as boxplots. ${ }^{* *} *$-value $=<0.001, p$-values were determined using a randomization test. (F, G) Cancer cells flowing in a microfluidic chamber, recorded live using a brightfield microscope and automatically tracked (Video 3). Examples of images used to train StarDist (F), and an example of results obtained using automated tracking are displayed $(\mathbf{G})$. The full tracks shown here were color-coded as a function of their $\times$ coordinate. 
the analysis pipeline presented here as it requires the manual annotations of the images to analyze (Extended data: Supplementary $\left.\operatorname{protocol}^{16}\right)$. For instance, to generate the training datasets presented in Figure 2, each cell/nuclei contour was drawn manually using the freehands selection tool in Fiji. The creation of a high-quality training dataset is a critical part of the process as it will impact the specificity and performance of the StarDist model. However, the generation of a training dataset is only required once per dataset type. If a StarDist model already exists for similar images it can be used to significantly accelerate the creation of the training dataset via semi-automated annotation (see Extended data: Supplementary $\left.\operatorname{protocol}^{16}\right)$.

One of our analysis pipeline's key features is that, once a StarDist model has been satisfactorily trained, movies of migrating cells can efficiently be processed in batch. Indeed, while individual tracking files can be analyzed one by one using the TrackMate graphical interface, we also provide a Fiji macro to analyze a folder containing multiple tracking files. Our batch processing macro will provide basic quantitative information for each track, including median and maximal speeds. If more information is needed, the tracking results generated by our script are directly compatible with the Motility lab website, where they can be further processed $^{17}$.

\section{Implementation and operation}

The described image analysis pipeline is composed of a Jupiter notebook optimized to run in Google Colab (ZeroCostDL4Mic framework ${ }^{15}$ ) and a Python script that can run in Fiji ${ }^{14}$. A step-by-step protocol describing how to use our analysis pipeline is provided as Extended data ${ }^{16}$.

\section{Use case}

To illustrate our analysis pipeline's functionality and flexibility, we first trained a StarDist model to analyze the behavior of breast cancer cells migrating collectively (Figure 2A; Extended data: Video $\left.1^{16}\right)$. The cancer cell's nuclei were fluorescently labeled, and the cells imaged using fluorescence-based microscopy. The creation of the training dataset (72 paired images, 24500 labelled objects) used in this example was greatly facilitated by the availability of a StarDist model, released by the StarDist creators, capable of segmenting fluorescent nuclei. In this case, the StarDist Fiji plugin was used to segment the location of nuclei in the training images, and all miss-annotations were manually corrected (Extended data: Supplementary protocol ${ }^{16}$ ). Of note, the workflow described here performs well in the presence of cell divisions as StarDist successfully recognise dividing cells, and TrackMate allows for track splitting.

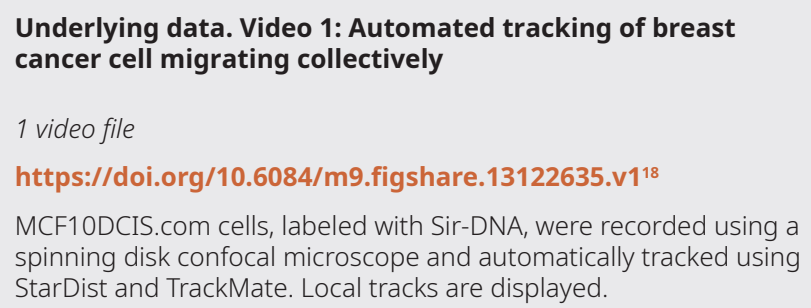

Cell migration experiments are typically performed using brightfield imaging. As it remains very challenging to segment brightfield objects using classical approaches, the field often relies on hand tracking to analyse cell migration movies. To highlight that our pipeline can also be used to analyze brightfield images, we generated a StarDist model (Training dataset after 5x augmentation: 209 paired images, 31200 labelled objects) to track $\mathrm{T}$ cells migrating on ICAM-1 or VCAM-1 (Figure 2C-E; Extended data: Video $2^{16}$ ). Importantly, automated analysis of these data could reproduce the results obtained via manual tracking ${ }^{19}$.

\section{Underlying data. Video 2: Automated tracking of T cell migrating on ICAM-1}

1 video file

https://doi.org/10.6084/m9.figshare.13122755.v120

Activated T cell plated ICAM-1 were recorded using a brightfield microscope and automatically tracked using StarDist and

TrackMate. Local tracks are displayed.

Finally, we used our pipeline to automatically track non-adherent cancer cells flowing in a microfluidic chamber (Figure 2F and G; Extended data: Video $3^{16}$ ). In this case, automated tracking is especially useful due to the very high number of frames to analyze (Training dataset: 57 paired images, 3680 labelled objects). For the last two examples, no suitable pre-trained StarDist models were available. Therefore, to generate the training datasets, we manually annotated 20 images and trained a first StarDist model. This model was then used to accelerate the annotation of the rest of the training images.

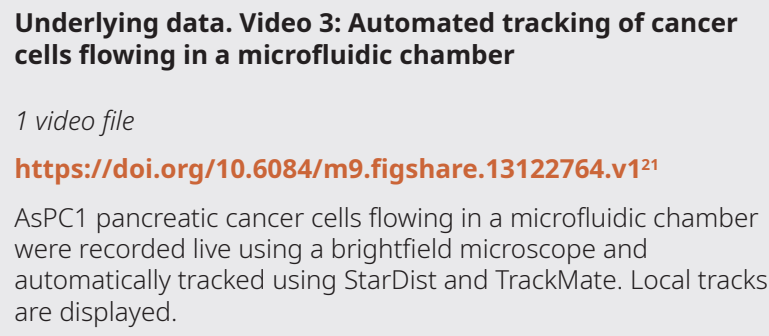

\section{Use case dataset creation}

Breast cancer cell dataset. MCF10DCIS.com cells were described previously ${ }^{15,22}$. DCIS.COM lifeact-RFP cells were incubated for $2 \mathrm{~h}$ with $0.5 \mu \mathrm{M}$ SiR-DNA (SiR-Hoechst, Tetu-bio, Cat Number: SC007) before being imaged live for $14 \mathrm{~h}$ using a spinning-disk confocal microscope (1 picture every $10 \mathrm{~min})$. The spinning-disk confocal microscope used was a Marianas spinning disk imaging system with a Yokogawa CSU-W1 scanning unit on an inverted Zeiss Axio Observer $\mathrm{Z} 1$ microscope (Intelligent Imaging Innovations, Inc.) equipped with a 20x (NA 0.8) air, Plan Apochromat objective (Zeiss).

T cell dataset. Lab-Tek 8 chamber slides (ThermoFisher) were coated with $2 \mu \mathrm{g} / \mathrm{mL}$ ICAM-1 or VCAM-1 overnight at $4^{\circ} \mathrm{C}^{19}$. Activated primary mouse $\mathrm{CD} 4+\mathrm{T}$ cells were washed 
and resuspended in L-15 media containing $2 \mathrm{mg} / \mathrm{mL}$ D-glucose. $\mathrm{T}$ cells were then added to the chambers, incubated $20 \mathrm{~min}$, gently washed to remove all unbound cells, and imaged. Imaging was done using a $10 \mathrm{x}$ phase contrast objective at $37^{\circ} \mathrm{C}$ on a Zeiss Axiovert 200M microscope equipped with an automated $\mathrm{X}-\mathrm{Y}$ stage and a Roper EMCCD camera. Time-lapse images were collected every $30 \mathrm{sec}$ for $10 \mathrm{~min}$ using SlideBook 6 software (Intelligent Imaging Innovations).

Flow chamber dataset. Cancer cells $(500,000$ cells $/ \mathrm{ml}$ in PBS) were perfused at a speed of $300 \mu \mathrm{m} / \mathrm{sec}$ using a peristaltic pump (ISMATEC MS12/4 analogic) and a homemade tubing system (Ismatek 3-Stop tubes and Ibidi ${ }^{\circledR}$ tubings and connectors) in a microchannel (Ibidi ${ }^{\circledR} \mu$-slides400 LUER). Images were acquired with a brightfield microscope (Zeiss Laser-TIRF 3 Imaging System, Carl Zeiss) and a 10X objective.

Data display and statistical analyses

Box plots were generated using PlotsOfData ${ }^{23}$. Randomization tests were performed using the online tool PlotsOfDifferences ${ }^{24}$.

\section{Conclusions}

Here we show that StarDist and TrackMate can be integrated seamlessly and robustly to automate cell tracking in fluorescence and brightfield images. We envision that this pipeline can also be applied to any circular or oval-shaped objects. However, we acknowledge that using brightfield images may not always work directly with our pipeline, especially if cells display complex and interchanging shapes, since StarDist is mostly designed to detect round or compact shapes. In this case, other tools, such as Usiigaci, could also be considered $^{8}$. Still, brightfield images could also be artificially labeled using deep learning, transforming the brightfield dataset into a pseudo-fluorescence one, as can be done with ZeroCostDL4Mic already ${ }^{15}$. The pipeline described here is currently limited to the tracking of objects in 2D. However, a similar workflow can be applied to 3D datasets as both StarDist and TrackMate can accommodate 3D images ${ }^{12,13,25}$.

\section{Data availability}

Underlying data

Zenodo: Combining StarDist and TrackMate example 1 - Breast cancer cell dataset, http://doi.org/10.5281/zenodo.403497626

Zenodo: Combining StarDist and TrackMate example 2 - T cell dataset, http://doi.org/10.5281/zenodo.403492927

Zenodo: Combining StarDist and TrackMate example 3 - Flow chamber dataset, http://doi.org/10.5281/zenodo.403493928
Extended data

Zenodo: Combining StarDist and TrackMate - Extended data, http://doi.org/10.5281/zenodo.4091467 ${ }^{16}$.

This project contains the following extended data:

- Supplementary protocol

- Video 1: Automated tracking of breast cancer cell migrating collectively. MCF10DCIS.com cells, labeled with Sir-DNA, were recorded using a spinning disk confocal microscope and automatically tracked using StarDist and TrackMate. Local tracks are displayed.

- Video 2: Automated tracking of $\mathbf{T}$ cell migrating on ICAM-1. Activated T cell plated ICAM-1 were recorded using a brightfield microscope and automatically tracked using StarDist and TrackMate. Local tracks are displayed.

- Video 3: Automated tracking of cancer cells flowing in a microfluidic chamber. AsPC1 pancreatic cancer cells flowing in a microfluidic chamber were recorded live using a brightfield microscope and automatically tracked using StarDist and TrackMate. Local tracks are displayed.

Data are available under the terms of the Creative Commons Attribution 4.0 International license (CC-BY 4.0).

\section{Software availability}

Source code available from: https://github.com/HenriquesLab/ ZeroCostDL4Mic

Archived source code at time of publication: http://doi.org/10.5281/ zenodo.4091474 ${ }^{29}$

License: MIT license.

Acknowledgments

The Cell Imaging and Cytometry Core facility (Turku Bioscience, University of Turku, Åbo Akademi University, and Biocenter Finland), the Finnish Euro-Bioimaging Node, and Turku Bioimaging are acknowledged for services, instrumentation and expertise.

This publication was supported by COST Action NEUBIAS (CA15124), funded by COST (European Cooperation in Science and Technology).
1. Jacquemet G, Baghirov H, Georgiadou M, et al.: L-type calcium channels regulate filopodia stability and cancer cell invasion downstream of integrin signalling. Nat Commun. 2016; 7: 13297.

PubMed Abstract | Publisher Full Text | Free Full Text
2. Jacquemet $\mathrm{G}$, Morgan MR, Byron A, et al.: Rac1 is deactivated at integrin activation sites through an IQGAP1-filamin-A-RacGAP1 pathway. J Cell SCi. 2013; 126(Pt 18): 4121-4135.

PubMed Abstract | Publisher Full Text | Free Full Text 
3. DuChez BJ: Automated Tracking of Cell Migration with Rapid Data Analysis. Curr Protoc Cell Biol. 2017; 76: 12.12.1-12.12.16.

PubMed Abstract | Publisher Full Text | Free Full Text

4. Cordelières FP, Petit V, Kumasaka M, et al.: Automated Cell Tracking and Analysis in Phase-Contrast Videos (iTrack4U): Development of Java Software Based on Combined Mean-Shift Processes. PLoS One. 2013; 8(11): e81266. PubMed Abstract | Publisher Full Text | Free Full Text

5. McQuin C, Goodman A, Chernyshev V, et al:: CellProfiler 3.0: Next-generation image processing for biology. PLOS Biol. 2018; 16(7): e2005970. PubMed Abstract | Publisher Full Text | Free Full Text

6. Piccinini F, Kiss A, Horvath P: CellTracker (not only) for dummies. Bioinformatics. 2016; 32(6): 955-957.

PubMed Abstract | Publisher Full Text

7. Barry DJ, Durkin $\mathrm{CH}$, Abella JV, et al.: Open source software for quantification of cell migration, protrusions, and fluorescence intensities. J Cell Biol. 2015; 209(1): 163-180.

PubMed Abstract | Publisher Full Text | Free Full Text

8. Tsai HF, Gajda J, Sloan TFW, et al.: Usiigaci: Instance-aware cell tracking in stain-free phase contrast microscopy enabled by machine learning. SoftwareX. 2019; 9: 230-237. Publisher Full Text

9. Pijuan J, Barceló C, Moreno DF, et al.: In vitro Cell Migration, Invasion, and Adhesion Assays: From Cell Imaging to Data Analysis. Front Cell Dev Biol. 2019; 7: 107.

PubMed Abstract | Publisher Full Text | Free Full Text

10. Chenouard N, Smal I, de Chaumont F, et al.: Objective comparison of particle tracking methods. Nat Methods. 2014; 11(3): 281-289. PubMed Abstract | Publisher Full Text | Free Full Text

11. Caicedo JC, Roth J, Goodman A, et al.: Evaluation of Deep Learning Strategies for Nucleus Segmentation in Fluorescence Images. Cytometry A. 2019; 95(9): 952-965.

PubMed Abstract | Publisher Full Text | Free Full Text

12. Tinevez JY, Perry N, Schindelin J, et al.: TrackMate: An open and extensible platform for single-particle tracking. Methods. 2017; 115: 80-90. PubMed Abstract | Publisher Full Text

13. Schmidt U, Weigert M, Broaddus C, et al.: Cell Detection with Star-Convex Polygons. In: Medical Image Computing and Computer Assisted Intervention - MICCAI 2018 (eds. Frangi, A. F., Schnabel, J. A., Davatzikos, C., Alberola-López, C. \& Fichtinger, G.) (Springer International Publishing, 2018). 11071: 265-273. Publisher Full Text

14. Schindelin J, Arganda-Carreras I, Frise E, et al.: Fiji: an open-source platform for biological-image analysis. Nat Methods. 2012; 9(7): 676-682. PubMed Abstract | Publisher Full Text | Free Full Text

15. Chamier LV, Laine RF, Jukkala J, et al.: ZeroCostDL4Mic: an open platform to use Deep-Learning in Microscopy. bioRxiv. 2020; 2020.03.20.000133. Publisher Full Text
16. Jacquemet G, Roy NH, Follain G, et al.: Combining StarDist and TrackMate - Extended data. Zenodo. 2020.

http://www.doi.org/10.5281/zenodo.4091467

17. Wortel IMN, Dannenberg K, Berry JC, et al.: CelltrackR: an R package for fast and flexible analysis of immune cell migration data. 2019. Publisher Full Text

18. Jacquemet G: Video 1: Automated tracking of breast cancer cell migrating collectively. f1000research.com. Media. 2020. http://www.doi.org/10.6084/m9.figshare.13122635.v1

19. Roy NH, Kim SHJ, Buffone A Jr, et al.: LFA-1 signals to promote actin polymerization and upstream migration in T cells. J Cell Sci. 2020; 133(17): jcs248328.

PubMed Abstract | Publisher Full Text | Free Full Text

20. Jacquemet G: Video 2: Automated tracking of T cell migrating on ICAM-1. f1000research.com. Media. 2020.

http://www.doi.org/10.6084/m9.figshare.13122755.v1

21. Jacquemet G: Video 3: Automated tracking of cancer cells flowing in a microfluidic chamber. f1000research.com. Media. 2020. http://www.doi.org/10.6084/m9.figshare.13122764.v1

22. Jacquemet $\mathrm{G}$, Paatero I, Carisey $A F$, et al.: FiloQuant reveals increased filopodia density during breast cancer progression. J Cell Biol. 2017; 216(10): 3387-3403.

PubMed Abstract | Publisher Full Text | Free Full Text

23. Postma M, Goedhart J: PlotsOfData-A web app for visualizing data together with their summaries. PLOS Biol. 2019; 17(3): e3000202. PubMed Abstract | Publisher Full Text | Free Full Text

24. Goedhart J: PlotsOfDifferences - a web app for the quantitative comparison of unpaired data. bioRxiv. 2019; 578575 .

Publisher Full Text

25. Weigert M, Schmidt U, Haase R, et al.: Star-convex Polyhedra for 3D Object Detection and Segmentation in Microscopy. 2020; 8 . Publisher Full Text

26. Jacquemet G: Combining StarDist and TrackMate example 1 - Breast cancer cell dataset (Version 1) [Data set]. Zenodo. 2020. http://www.doi.org/10.5281/zenodo.4034976

27. Roy NH, Jacquemet G: Combining StarDist and TrackMate example $\mathbf{2}$ - T cell dataset (Version 1) [Data set]. Zenodo. 2020. http://www.doi.org/10.5281/zenodo.4034929

28. Follain G, Jacquemet G: Combining StarDist and TrackMate example 3 - Flow chamber dataset (Version 1) [Data set]. Zenodo. 2020 http://www.doi.org/10.5281/zenodo.4034939

29. Jacquemet G, Fazeli E, Laine RF, et al.: Combining StarDist and TrackMate - Archived source code (Version v1). Zenodo. 2020. http://www.doi.org/10.5281/zenodo.4091474 


\section{Open Peer Review}

\section{Current Peer Review Status:}

\section{Version 2}

Reviewer Report 23 December 2020

https://doi.org/10.5256/f1000research.31313.r76337

(C) 2020 Arganda-Carreras I. This is an open access peer review report distributed under the terms of the Creative Commons Attribution License, which permits unrestricted use, distribution, and reproduction in any medium, provided the original work is properly cited.

\section{Ignacio Arganda-Carreras}

Department of Computer Science and Artificial Intelligence, Basque Country University, San Sebastian, Spain

I have no further comments to make. The authors have properly addressed everything I mentioned in my previous review.

Competing Interests: No competing interests were disclosed.

Reviewer Expertise: Computer Vision, Bioimage Analysis.

I confirm that I have read this submission and believe that I have an appropriate level of expertise to confirm that it is of an acceptable scientific standard.

\section{Version 1}

Reviewer Report 16 November 2020

https://doi.org/10.5256/f1000research.29845.r73862

(c) 2020 Swaminathan V. This is an open access peer review report distributed under the terms of the Creative Commons Attribution License, which permits unrestricted use, distribution, and reproduction in any medium, provided the original work is properly cited.

\section{Vinay Swaminathan}

1 Lund University, Lund, Sweden

2 Lund University, Lund, Sweden

In this article, Fazeli et al. combine deep-learning segmentation tools with open access cell 
tracking platforms to show the feasibility for automated cell tracking and large population motility analysis. Using this approach, the authors here clearly describe and validate this process in fluorescent movies of collective cell migration as well as single cell bright-field cell migration datasets. While the description of methods is very clear (and special kudos to the authors for transparency and availability), and its applicability very apparent, a few other potential points to consider are as follows:

1. A comparison on a given dataset between trackmate with StarDist based segmentation versus canonical segmentation methods of the nuclei. How much better does it perform? This could be in terms of accuracy/precision or time taken to analyze.

2. Related to point 1: The power of StarDist based nuclear segmentation is its ability in crowded environments as well as where there is poor signal in the fluorescent channels. Is there a way to explicitly show that when there is poor signal or bleaching during a movie, StarDist combined with trackmate does a better job?

3. One of the hardest objects to segment are bright-field objects which is why the field has been relying on hand tracking of cell migration movies taken with bright-field. I think the authors should underscore this and highlight that this method is overcoming this big challenge enabling larger population analysis.

Is the rationale for developing the new software tool clearly explained?

Yes

Is the description of the software tool technically sound?

Yes

Are sufficient details of the code, methods and analysis (if applicable) provided to allow replication of the software development and its use by others?

Yes

Is sufficient information provided to allow interpretation of the expected output datasets and any results generated using the tool?

Yes

Are the conclusions about the tool and its performance adequately supported by the findings presented in the article?

Yes

Competing Interests: No competing interests were disclosed.

Reviewer Expertise: Cell migration, mechanobiology, Cancer.

I confirm that I have read this submission and believe that I have an appropriate level of expertise to confirm that it is of an acceptable scientific standard.

Author Response 08 Dec 2020 


\section{Guillaume Jacquemet}

We thank the reviewer for his positive comments. Regarding the performance of Deep Learning and StarDist for nuclear segmentation, this topic has been extensively covered by others, and I would recommend the excellent paper from Caicedo et al. (Cytometry, 2019). In addition, StarDist was demonstrated to perform very well on images containing dividing cells, extensive noise and nuclei deformation. This has also been our experience. We would also add that the main reason why we started to use StarDist was that we could not segment our cell migration movies with enough accuracy using intensity-based thresholding. Because of this issue, automated tracking was not feasible. We would argue that the best way to ensure that the results generated by automated tracking approach are of good quality are 1) the visual inspection of the tracks and 2) comparing the results obtained compared to manual tracking.

We fully agree with the reviewer that the automatic tracking of brightfield movies is very challenging and that the field often relies on manual analysis. We have now added a few sentence to the text to highlight this further. We indeed hope that the protocol published here may help to alleviate some of this burden.

Competing Interests: No competing interests were disclosed.

Reviewer Report 11 November 2020

https://doi.org/10.5256/f1000research.29845.r73865

(C) 2020 Arganda-Carreras I. This is an open access peer review report distributed under the terms of the Creative Commons Attribution License, which permits unrestricted use, distribution, and reproduction in any medium, provided the original work is properly cited.

\section{Ignacio Arganda-Carreras}

${ }^{1}$ Department of Computer Science and Artificial Intelligence, Basque Country University, San Sebastian, Spain

2 Department of Computer Science and Artificial Intelligence, Basque Country University, San Sebastian, Spain

The authors propose the combination of open-source tools (namely StarDist and TrackMate) for the automatic tracking of cells in fluorescence and brightfield images in 2D. Moreover, they provide a step-by-step workflow to process videos in a batch mode using exclusively free tools. They evaluate its performance by comparing the results obtained using such a workflow with those of manual tracking from an already published public dataset.

The paper is very well written, concise and straight to the point, with a special emphasis on reproducibility.

As pointed out in the conclusions, one of the limitations of the proposed approach is inherent to 
the type of objects that StarDist can properly segment (basically round). However, that doesn't prevent this pipeline from being extremely useful for a broad spectrum of cell tracking problems. Moreover, the adaptation of the pipeline to 3D images seems pretty straightforward.

Something interesting that is not mentioned in the paper is how well the workflow would perform in the presence of cell divisions of apoptosis. That could be easily tested using some of the datasets from the Cell Tracking Challenge (http://celltrackingchallenge.net/2d-datasets/).

Minor comments:

Please homogenize how you write "deep learning", which appear sometimes as "DeepLearning" and sometimes as "deep learning".

Is the rationale for developing the new software tool clearly explained?

Yes

Is the description of the software tool technically sound?

Yes

Are sufficient details of the code, methods and analysis (if applicable) provided to allow replication of the software development and its use by others?

Yes

Is sufficient information provided to allow interpretation of the expected output datasets and any results generated using the tool?

Yes

Are the conclusions about the tool and its performance adequately supported by the findings presented in the article?

Yes

Competing Interests: No competing interests were disclosed.

Reviewer Expertise: Computer Vision, Bioimage Analysis.

I confirm that I have read this submission and believe that I have an appropriate level of expertise to confirm that it is of an acceptable scientific standard, however I have significant reservations, as outlined above.

Author Response 08 Dec 2020

\section{Guillaume Jacquemet}

We thank the reviewer for his positive comments. The reviewer highlights an excellent point when asking about the ability of the pipeline to cope with cell division. This is indeed a critical concern when tracking cells for an extended period of time. Numerous cell divisions were actually detected in our DCIS.com test dataset. In this case, our pipeline worked very well, and division events were both recognized, and the tracks splits after divisions. This is 
due to two factors 1) we trained the StarDist model also to recognize mitotic cells and 2) we enabled track splitting in TrackMate. We have now added a sentence in the manuscript to reflect this.

Competing Interests: No competing interests were disclosed.

Reviewer Report 11 November 2020

https://doi.org/10.5256/f1000research.29845.r73864

(C) 2020 Whitehead L. This is an open access peer review report distributed under the terms of the Creative Commons Attribution License, which permits unrestricted use, distribution, and reproduction in any medium, provided the original work is properly cited.

\section{Lachlan W. Whitehead}

1 The Walter and Eliza Hall Institute of Medical Research, Parkville, Vic, Australia

2 The Walter and Eliza Hall Institute of Medical Research, Parkville, Vic, Australia

This article presents a pipeline for analyzing cell migration in a variety of contexts by combining several complimentary techniques. Utilising stardist for cell detection (provided cells are round/have nuclei staining), and trackmate for connecting the detected nuclei over time - a startto-finish protocol is described allowing a microscopist with little image analysis knowledge to be able to quantify their experiment.

The authors do an admirable job of describing the required steps of the analysis pipeline, including an introduction to Jupyter Notebooks and the ZeroCostDL4Mic workflows to train a custom Stardist model. They also provide a FIJI macro for batch analysis, potentially saving a researcher many hours of human analysis time.

Indeed, as all of these methods are published and validated already my only (small) criticism has to do with the training of the stardist models. As the authors rightly note, this is the most time consuming part of the analysis and as a result I would have liked to see some mention of how much manual time is required. For instance, while the article suggests training a small 20 image dataset and then using transfer learning to speed up the remaining annotation, the number of remaining images is not described. While the amount of training required is likely to vary across experiments, I think readers would benefit from knowing when considering this pipeline how many cells (rather than image fields of view) they are likely to be required to manually annotate. Perhaps for each dataset where a model was trained the authors could specify the size of the training dataset (in both images and number of cells).

Overall, this is a clear description of several powerful tools being combined into a very useful and versatile workflow.

Is the rationale for developing the new software tool clearly explained? 
Yes

Is the description of the software tool technically sound?

Yes

Are sufficient details of the code, methods and analysis (if applicable) provided to allow replication of the software development and its use by others?

Yes

Is sufficient information provided to allow interpretation of the expected output datasets and any results generated using the tool?

Yes

Are the conclusions about the tool and its performance adequately supported by the findings presented in the article?

Yes

Competing Interests: No competing interests were disclosed.

Reviewer Expertise: bioimage analysis

I confirm that I have read this submission and believe that I have an appropriate level of expertise to confirm that it is of an acceptable scientific standard.

Author Response 08 Dec 2020

\section{Guillaume Jacquemet}

We thank the reviewer for his positive comments. The reviewer makes an excellent point. We have now added in the main text of the manuscript the size of the training dataset, including both the number of images as well as the total number of annotated cells. The training dataset size are as follow: Breast cancer cell dataset ( 72 paired images, 24500 labelled objects); T cell dataset (Training dataset after 5x augmentation: 209 paired images, 31200 labelled objects); Flow chamber dataset (Training dataset: 57 paired images, 3680 labelled objects). We estimate that it takes between 4-8h of work to annotate a completely new training dataset.

Competing Interests: No competing interests were disclosed. 
The benefits of publishing with F1000Research:

- Your article is published within days, with no editorial bias

- You can publish traditional articles, null/negative results, case reports, data notes and more

- The peer review process is transparent and collaborative

- Your article is indexed in PubMed after passing peer review

- Dedicated customer support at every stage

For pre-submission enquiries, contact research@f1000.com 